\title{
AVALIAÇÃO DA QUALIDADE DE COMPRIMIDOS DE CLORIDRATO DE AMITRIPTILINA DISPENSADOS NA REDE PÚBLICA DA CIDADE DE ITACOATIARA - AM
}

\author{
C. S. B. AGUILERA ${ }^{1}$, N. S. MENDONÇA ${ }^{2}$, S. F. OLIVEIRA ${ }^{2}$, S. P. M. COSTA ${ }^{3}$, P. J. \\ ROLIM NETO $^{2}$, K. E. R. SILVA ${ }^{2}$ \\ ${ }^{1}$ Universidade Federal de Pernambuco, Laboratório de Tecnologia de Medicamentos \\ ${ }^{2}$ Universidade Federal do Amazonas, Laboratório de Tecnologia Farmacêutica \\ ${ }^{3}$ Universidade Federal de Sergipe, Departamento de Farmácia
}

E-mail para contato: ramos.keyla@gmail.com

\begin{abstract}
RESUMO - A depressão é um importante problema de saúde global, causando impacto negativo na vida dos indivíduos e suas famílias. Representante da classe dos antidepressivos triciclicos, a amitriptilina é o antidepressivo mais consumido pelos usuários da rede pública de saúde no município de Itacoatiara/AM. O objetivo deste trabalho foi avaliar a qualidade de comprimidos contendo cloridrato de amitriptilina, distribuídos na rede pública do município, através dos testes de análise visual, peso médio, dureza, tempo de desintegração, dissolução e doseamento. Os comprimidos revestidos, apresentaram coloração amarela uniforme, superficie lisa sem manchas, sem rachaduras, fissuras ou trincas, a embalagem apresentou-se legivel e sem nenhum comprimido faltante. Quanto ao peso médio, os limites de variação para o comprimido do laboratório A e B foram de 1,05\% e 1,53\% respectivamente. A dureza média foi de $36 \mathrm{~N}$ e $34 \mathrm{~N}$ para os comprimidos do laboratório A e $B$ respectivamente. As amostras desintegraramse em tempo inferior a 15 minutos, no teste de dissolução, obteve-se valores abaixo de $95 \%$ de substância ativa dissolvida no meio de dissolução e no ensaio de doseamento, obteve-se teores de 99 e 100\%. Concluindo-se então que as duas amostras de comprimidos analisadas, foram aprovadas cumprindo os requisitos dos testes preconizados pela Farmacopeia Brasileira, $5^{a}$ edição, apresentando propriedades que as qualificaram como um produto adequado para o consumo, podendo ser utilizadas pela população.
\end{abstract}

Palavras-Chave: controle de qualidade, comprimidos, antidepressivos tricíclicos, cloridrato de amitriptilina

\footnotetext{
ABSTRACT - Depression is an important global health problem, causing a negative impact on the lives of individuals and their families. Representative of the class of tricyclic antidepressants, amitriptyline is the antidepressant most consumed by users of the public health network in the municipality of Itacoatiara / AM. The objective of this study was to
} 
evaluate the quality of tablets containing amitriptyline hydrochloride, distributed in the public network of the city, through the tests of visual analysis, mean weight, hardness, disintegration time, dissolution and dosing. The coated tablets showed uniform yellow coloration, smooth surface without blemishes, without cracks, cracks or cracks, the package was legible and without any missing tablets. Regarding the mean weight, the limits of variation for the tablet of laboratory $A$ and $B$ were of $1,05 \%$ and $1,53 \%$ respectively. The mean hardness was $36 N$ and 34 $N$ for laboratory tablets $A$ and $B$ respectively. Samples disintegrated in less than 15 minutes in the dissolution test, values above 95\% of active substance dissolved in the dissolution medium were obtained and in the assay assay, contents of 99 and $100 \%$ were obtained. It was concluded that the two tablets analyzed were approved to meet the requirements of the tests recommended by the Brazilian Pharmacopoeia, 5th edition, presenting properties that qualified them as a suitable product for consumption, and could be used by the population.

Keywords: quality control, tablet, tricyclic antidepressants, amitriptyline hydrochloride

\section{INTRODUÇÃ̃O}

Milhões de pessoas sofrem algum tipo de doença mental no mundo e esse número tem aumentado de modo progressivo, principalmente nos países em desenvolvimento. A depressão é um transtorno afetivo caracterizado por uma alteração psíquica e orgânica com consequentes modificações na maneira de avaliar a realidade e a vida (TOLEDO et al, 2005), causando impacto negativo na vida dos indivíduos e de suas famílias, além de gerar altos custos para o sistema de saúde e para a sociedade (OMS, 2002; MOLINA et al. 2012).

O tratamento farmacológico dos quadros depressivos geralmente se faz com o uso de antidepressivos (GOODMAN \& GILMAN, 2005). Medicamentos que ajudam a restaurar o equilíbrio químico no cérebro, aumentando a concentração de neurotransmissores na sinapse no sistema nervoso central (SNC) (TOLEDO et al. 2005). Os antidepressivos têm sido utilizados em larga escala na rede pública do município de Itacoatiara, Amazonas, Brasil, especialmente a amitriptilina, um dos representantes da classe dos antidepressivos tricíclicos, uma substância sujeita à receita de controle especial em duas vias, constando na lista C1 (outras substâncias sujeitas a controle especial) (BRASIL, 2010a; BRASIL, 1998; BRASIL, 2010b).

Segundo dados da Secretaria Municipal de Saúde (SEMSA) do município de Itacoatiara, a amitriptilina é o medicamento antidepressivo mais consumido no município pelos pacientes da rede pública de saúde, em segundo lugar está a fluoxetina, um antidepressivo da classe dos Inibidores Seletivos da Recaptação da Serotonina (ISRS). No ano de 2015, o consumo de amitriptilina foi de 30.310 comprimidos, no ano de 2016, no período de janeiro a junho foi de 17.220 comprimidos, estando entre o terceiro mais consumido.

O controle de qualidade é um conjunto de medidas destinadas a verificar a qualidade de cada lote de medicamentos, objetivando verificar se o produto está em conformidade com as especificações farmacopéicas. Este é um requisito das Boas Práticas de Fabricação (BPF) e é executado em todas as etapas de produção do medicamento, a 
fim de evitar falhas e detectar desvios da qualidade do produto que possam implicar em riscos ao paciente (BRASIL, 2010c; GIL, 2010; RIGOBELLO et al.2013).

Face ao exposto, visto que, a amitriptilina é um antidepressivo amplamente distribuído pela rede pública do município de Itacoatiara e a falta de qualidade do medicamento pode ocasionar o comprometimento da saúde dos pacientes, o objetivo deste trabalho foi avaliar a qualidade de comprimidos contendo cloridrato de amitriptilina, distribuídos na rede pública do município de Itacoatiara, sendo esses de laboratórios farmacêuticos diferentes, um medicamento genérico e um medicamento similar, através dos testes de análise visual, peso médio, dureza, tempo de desintegração, dissolução e doseamento.

\section{MATERIAIS E MÉTODOS}

Os comprimidos utilizados foram: cloridrato de amitriptilina $25 \mathrm{mg}$, genérico Lote: 2444817 do laboratório A e similar - Lote: 15064815 do laboratório B, sendo assim denominados como amostras do laboratório A e B. Para realização dos testes de controle de qualidade utilizou-se 160 comprimidos. Os lotes foram adquiridos gratuitamente pela SEMSA/ CAF (Central de Abastecimento Farmacêutico) do município de Itacoatiara/Amazonas. Utilizou-se padrão de trabalho de amitriptilina, com teor de pureza de $99 \%$.

O aspecto visual e as análises de determinação de peso médio e desintegração foram realizadas no LTF do Instituto de Ciências Exatas e Tecnologia - ICET/UFAM. Os ensaios de dureza, dissolução e doseamento foram realizados no LTM.

$\mathrm{O}$ aspecto visual das amostras foram analisadas quanto à uniformidade de coloração dos comprimidos, revestimento, comprimidos faltantes, quebrados ou trincados, legibilidade na embalagem e qualquer outra alteração aparente (BIANCHIN et al. 2010; GIL, 2010). As análises de determinação do peso médio, dureza, teste de desintegração, teste de dissolução e doseamento por espectrofotometria de absorção no ultravioleta, foram realizadas de acordo com as especificações descritas na Farmacopéia Brasileira $5^{\text {a }}$ edição, volume 1 e 2 .

\section{RESULTADOS E DISCUSSÃO}

A análise visual no que diz respeito a medicamento é um ensaio de qualidade cuja finalidade principal é avaliar integridade física e estética do produto (GIL, 2010). O aspecto geral de um comprimido e a sua identidade visual, são características essenciais para que seja aceito pelo doente, para o controle de uniformidade entre comprimidos e, para assegurar a inexistência de problemas (LACHMAN et al. 2001).

Os comprimidos de cloridrato de amitriptilina $25 \mathrm{mg}$, são comprimidos revestidos, apresentaram coloração amarela uniforme, superfície lisa sem manchas, sem rachaduras, fissuras ou trincas. A embalagem apresentou-se legível e sem nenhum comprimido faltante.

O ensaio de determinação do peso médio dos comprimidos de cloridrato de amitriptilina $25 \mathrm{mg}$ do laboratório A e B, tem seu resultados apresentados na Tabela 2. 
Tabela 2 - Valores obtidos na determinação de peso de comprimidos de Amitriptilina 25 mg.

\begin{tabular}{ccc}
\hline & & Peso $(\mathbf{g})$ \\
\hline Amostra & Laboratório A & Laboratório B \\
$\sum^{\mathbf{1}} \mathbf{( g )}$ & 2,46 & 3,455 \\
Média $(\mathbf{g})$ & 0,123 & 0,17275 \\
Máximo & 0,132225 & 0,18570625 \\
Mínimo $^{\mathbf{2}} \mathbf{( g )}$ & 0,113775 & 0,15979375 \\
$\mathbf{D P}^{\mathbf{3}} \mathbf{( \% )}$ & 0,0012977 & 0,00265016 \\
$\mathbf{C V}^{\mathbf{1}}$ & 1,05 & 1,53 \\
Resultado & Aprovado & Aprovado
\end{tabular}

$\sum^{1}=$ Somatório; $\mathrm{DP}^{2}=$ Desvio Padrão; $\mathrm{CV}^{3}=$ Coeficiente de Variação.

Segundo a Farmacopéia Brasileira, para comprimidos com peso médio de mais que $80 \mathrm{mg}$ e menos que $250 \mathrm{mg}$, o limite de variação permitido é de $\pm 7,5 \%$. Observouse que todos os comprimidos submetidos ao teste estão dentro dos limites especificados em relação ao peso médio, demonstrando assim homogeneidade de peso, boa mistura, bom fluxo, baixa aderência e adequada força de compressão. O que demonstra que o processo de fabricação, no quesito peso médio encontra-se adequado sugerindo que houve correto preenchimento da matriz e regulagem dos punções superior e inferior (MOISÉS, 2006).

A dureza de um comprimido é proporcional à força de compressão, inversamente proporcional à sua porosidade (BRASIL, 2010e) e visa demonstrar a resistência dos comprimidos ao esmagamento ou à ruptura sob pressão radial. Considerada uma característica física muito importante para comprimidos, ela garante a integridade desta forma farmacêutica, permitindo que ele suporte os choques mecânicos nos processos de revestimento, drageamento, embalagem e transporte (FERRAZ et al. 1998).

O teste de dureza foi determinado utilizando dez comprimidos, do laboratório A e do laboratório B em Newton (unidade de força). A dureza média para os comprimidos do laboratório A e B foram respectivamente $36 \mathrm{~N}$ e $34 \mathrm{~N}$, estando em conformidade com o preconizado pela Farmacopéia Brasileira (2010e), que determina que nenhuma unidade apresente dureza inferior a $30 \mathrm{~N}$.

A desintegração de comprimidos pode influenciar na absorção, na biodisponibilidade e na ação terapêutica do fármaco. Para que o princípio ativo de comprimidos de liberação imediata seja absorvido e exerça a sua ação farmacológica rapidamente, é necessário que ocorra a desintegração adequada do comprimido em partículas menores, favorecendo, portanto, a absorção e a biodisponibilidade adequada do fármaco no organismo (PEIXOTO et al. 2005). A não desintegração dos comprimidos no tempo especificado, pode fazer com que eles sejam eliminados da forma como foram ingeridos, não sendo absorvidos pelo organismo e não produzindo o efeito terapêutico esperado (MOISÉS, 2006).

A desintegração dos comprimidos do laboratório A ocorreu em 6 minutos e do laboratório B desintegraram-se em 14 minutos. A diferença no tempo de desintegração pode ser explicada pela composição de cada amostra, visto que os comprimidos do laboratório A apresentam em sua formulação croscarmelose sódica, um derivado sódico da celulose, que demonstra um intumescimento quando em contato com água, podendo aumentar seu volume inicial de quatro a oito vezes, gerando um grande poder de desintegração (FERRERO et al. 1997). Já o comprimido do laboratório B não apresenta esse excipiente em sua formulação, desintegrando-se em tempo maior.

De acordo com a Farmacopéia Brasileira $5^{\mathrm{a}}$ edição, volume 2, o limite máximo de tempo para que ocorra a desintegração total de todos os comprimidos do ensaio é de 
15 minutos. As amostras analisadas no presente trabalho cumprem o teste, indicando que, frente a força de compressão empregada, o desintegrante é capaz de atuar quando em contato com os fluidos biológicos, desintegrando os comprimidos.

A dissolução dos comprimidos está relacionada com a biodisponibilidade do fármaco no organismo. Em vista disso, é imprescindível que os comprimidos sejam dissolvidos, liberando

uma determinada porcentagem do princípio ativo para o meio de dissolução, a fim de que o mesmo torne-se disponível para exercer a sua ação farmacológica (PEIXOTO et al. 2005).

Os resultados do teste de dissolução das duas amostras analisadas encontram-se na Tabela 3. De acordo com o procedimento metodológico o valor mínimo aceitável de cloridrato de amitriptilina dissolvido em 45 minutos, no meio de dissolução é de 75\% (Q) da quantidade declarada de $\mathrm{C}_{20} \mathrm{H}_{23} \mathrm{~N}$.HCl (BRASIL,2010). Portanto, os comprimidos de cloridrato de amitriptilina analisados encontram-se não conformes com as especificações estabelecidas, visto que, obteve-se valores dentro da faixa especificada de substância ativa dissolvida no meio de dissolução.

Tabela 3 - Resultados do ensaio de dissolução de comprimidos de cloridrato de amitriptilina $25 \mathrm{mg}$.

\begin{tabular}{ccccc} 
& LABORATÓRIO & \multirow{2}{*}{ LABORATÓRIO } & \multirow{2}{*}{ [ ]\% } \\
\hline $\mathbf{1}$ & $\mathbf{A}$ & & $\mathbf{B}$ & 93,446 \\
$\mathbf{2}$ & 0,399 & 96,844 & 0,385 & 104,126 \\
$\mathbf{3}$ & 0,366 & 88,834 & 0,429 & 96,601 \\
Média & 0,411 & 99,757 & 0,398 & 98,058 \\
DP $^{1}$ & 0,392 & 95,145 & 0,404 & 5,486
\end{tabular}

$\mathrm{DP}^{1}=$ Desvio Padrão.

Após a análise com Teste $T$, o t calculado foi de 0,684283 e o t tabelado de 4,30265, logo, não existe diferença significante e o nível de confiança entre o medicamento do laboratório A e o medicamento do laboratório B é de $95 \%$.

O doseamento dos fármacos é de extrema importância na avaliação da qualidade dos produtos farmacêuticos, visto que determina a quantidade de princípio ativo presente na formulação a ser administrada no organismo (ZARBIELL, 2007; GIL, 2010).

A administração de um medicamento com concentração de princípio ativo acima da concentração declarada na fórmula pode representar um sério risco de intoxicação para o paciente. Por outro lado, o medicamento com um teor de princípio ativo abaixo da concentração indicada na fórmula do produto resultará em falha terapêutica, comprometendo o quadro clínico do usuário do medicamento (PEIXOTO, et al. 2005).

No presente trabalho o teor de cloridrato de amitriptilina foi determinado em triplicata por método espectrofotométrico conforme a monografia do comprimido de cloridrato de amitriptilina presente na Farmacopéia Brasileira, 5a edição, volume 2. A quantidade declarada da substância deve conter no mínimo $90 \%$ e no máximo $110 \%$. No ensaio de doseamento, obteve-se teores de 99 e $100 \%$, inferiores a $110 \%$ e superiores a $90 \%$, concluindo-se então que o comprimido cumpre teste de doseamento. As médias das absorbâncias obtidas estão descritas na Tabela 4. 
Tabela 4 - Resultados do ensaio de doseamento por espectrofotometria de absorção no ultravioleta.

$\begin{array}{ccc}\text { AMOSTRA } & \text { VALORES DE } & \text { TEOR [\%] } \\ \text { ABSORBÂNCIAS } & 100 \% \\ \text { Padrão } & 0,412 & 99 \% \\ \text { Laboratório A } & 0,411 & 100 \%\end{array}$

\section{CONCLUSÃO}

A qualidade dos medicamentos produzidos pelos dois laboratórios foi evidenciada pelo ensaio, o que garantirá a eficácia e a segurança da população usuária.

\section{REFERENCIAS}

ALEIXO, F. C.; MARQUES, S. DE A.; SAMPAIO, P. G. Estudo da metodologia para quantificação de cloridrato de amitriptilina em cápsulas manipuladas em diferentes farmácias de manipulação de Jundiaí - SP. Rev. Multidisciplinar da Saúde, n. 07, p. 47-64, 2012.

BIANCHIN, M. D. et al. Avaliação da qualidade de comprimidos de propranolol e enalapril distribuídos no sistema público de saúde em uma cidade do sul do Brasil. Ciênc. saúde coletiva. Rio de Janeiro. Vol. 17, n. 2 (2012), p. 491-498, 2012.

BRASIL. Farmac. Bras.. v. 1 vol. Brasília: Anvisa, 2010e. 524 p.

BRASIL. Farmac. Bras.. v. 2. Brasília: Anvisa, 2010d. 808 p.

BRASIL. RDC n⿳ 21 , de 17 de junho de 2010b. Dispõe sobre a atualização do Anexo I, Listas de Substâncias Entorpecentes, Psicotrópicas, Precursoras e Outras sob Controle Especial, da Portaria SVS/MS n 344, de 12 de maio de 1998, e dá outras providências. Disponível em: <http://brasilsus.com.br/legislacoes/rdc/104438-21.html>. Acesso em: 06 de ago. 2016.

BRASIL. Resolução 17, de 16 de abril de 2010. Dispõe sobre as Boas Práticas de Fabricação de Medicamentos. Diário Oficial da União, Poder Executivo, Brasília, DF, 06 de abril de 2010c. Disponível em: $<$ http://bvsms.saude.gov.br/bvs/saudelegis/anvisa/2010/res0017_16_04_2010.html>. Acesso em 06 de ago. 2016.

FERRAZ, G. H.; CONSIGLIERI, V. O.; STORPIRTIS, S. Avaliação da cinética de dissolução de ampicilina em comprimidos comercializados no Brasil. Rev. Farm. Bioq. Univ. São Paulo, v. 34, n. 2, p. 93-99, 1998.

FERRERO, C.; JIMÉNEZ-CASTELLANOS, M. R. The influence of carbohydrate nature and drying methods on the compaction properties and pore structure of new methyl methacrylate copolymers. Int. J. Pharm. v. 248, n. 1, p. 157-171, 2002. 
GIL, E. S. Controle Físico-Químico de Qualidade de Medicamentos, $3^{\mathrm{a}}$ ed. São Paulo: Pharmabooks, 2010. p. 512 p.

GOODMAN \& GILMAN, As Bases Farmacológicas da Terapêutica. 10a ed., Rio de Janeiro: Mc Graw Hill, 2003. 1647 p.

LACHMAN, L.; LIEBERMAN, H.A.; KANING, J.L. Teoria e Prática na Indústria Farmacêutica. Lisboa: Fundação Calouste, 2001. 1517 p.

MOISÉS, P. R. Tecnologia de Produção de Comprimidos. Fármacos e Medicamentos, São Paulo, v, 7, n.38, p.38-46, janeiro de 2006. Disponível em: $<$ www.conectepharma.com.br/artigos/ down.asp?COD=244> . Acesso em: 02 de set. 2016.

MOLINA, M. R. A. et al. Prevalência de depressão em usuários de unidades de atenção primária. Psiq. Clín., v. 39, n. 6, p. 194-7.

ORGANIZAÇÃO MUNDIAL DA SAÚDE. Relatório mundial de saúde: Saúde mental: nova concepção, nova esperança. 1. ${ }^{a}$ ed., Lisboa: OMS, 2002.

PEIXOTO, M. M. et al. Avaliação da qualidade de comprimidos de captopril dispensados em Feira de Santana-BA. Infarma, v. 16, n. 13-14, p. 69-73, 2005.

RANG, H. P.; DALE, M. M.; RITTER, J. M. Farmacologia. $6^{\text {a }}$ ed., Rio de Janeiro: Elsevier, 2011. 904 p.

RIGOBELlO, C.; GASPARETTO, A. V.; DINIZ, A.; RABITO, M. F.; NERY, A. A. F. Avaliação da qualidade e perfil de dissolução de comprimidos de cloridrato de propranolol. Acta sci., Health sci., v. 35, n. 1, p. 85-90, 2013.

TEODORO, W. L. G. Depressão: corpo, mente e alma. $3^{\text {a }}$ ed., Wagner Luiz Garcia Teodoro, 2010. $240 \mathrm{p}$.

TOLEDO, R. A. et al. Estudo eletroquímico e químico-quântico da oxidação do antidepressivo tricíclico amitriptilina. Quím. Nova, v. 28, n. 3, p. 456, 2005.

WORLD HEALTH ORGANIZATION. Depression. 2016. Disponível em < http://www.who.int/mediacentre/factsheets/fs369/en/ > Acesso: 02 de set. 2016.

ZARBIELLI, M. G.; MACEDO, S. M. D; MENDEZ, A. S. L. Controle de qualidade de cápsulas de piroxicam manipuladas em farmácias do município de Erechim-RS. Rev. Bras. Farm., v. 87, n. 2, p. 55-59, 2006.

\section{AGRADECIMENTOS}

A Secretaria Municipal de Saúde do município de Itacoatiara/AM, pela doação dos comprimidos utilizados nas análises. Ao Instituto de Ciências Exatas e Tecnologia/UFAM pela utilização das instalações e equipamentos. Ao Laboratório de Tecnologia de Medicamento/UFPE, pela pareceria e pela utilização das instalações e equipamentos. 